\title{
Heartbeat: Intervention for spontaneous coronary artery dissection?
}

\author{
doi:10.1136/heartjnl-2021-320055
}

Catherine M Otto

Acute coronary syndrome due to spontaneous coronary artery dissection (SCAD) typically is managed conservatively because coronary anatomy returns to normal in most patients at follow-up and because there is a high rate of technical failure and complications with percutaneous coronary intervention (PCI). However, a subgroup of these patients is at risk of extensive myocardial infarction and might benefit from PCI, despite the risk of complications. In this issue of Heart, Kotecha and colleagues ${ }^{1}$ report outcomes in 215 SCAD patients (94\% women, median age 48 years with a range of 42-54 years) who underwent PCI for ST-elevation myocardial infarction, cardiac arrest, TIMI grade $0 / 1$ flow or proximal dissection (figure 1). As expected, PCI-related complications were frequent, occurring in 38.6\% (83/215), with $13.0 \%(28 / 215)$ of patients suffering a serious complication. Even so, improved TIMI flow was achieved in $84.3 \%$ $(118 / 140)$ with worsened TIMI flow in only $7 \%$ of patients. At a median follow-up of 900 days , 9.3\% suffered recurrent acute myocardial infarction, $4.7 \%$ required further revascularisation, $1.5 \%$ had a stroke and 1.4\% died. However, there was no difference in outcomes in SCAD-PCI patients compared with a matched cohort of SCAD patients who did not undergo PCI.

Adamson $^{2}$ reminds us that SCAD may account for as many as $2 \%-3 \%$ of acute coronary syndromes and predominantly affects middle-aged women without traditional risk factors for coronary disease. $\mathrm{He}$ also points out that in the study by Kotecha and colleagues ${ }^{1}$ the SCAD patients undergoing PCI, compared with those treated conservatively, more often suffered myocardial infarction or cardiac arrest and more often had left main or proximal left anterior descending coronary artery involvement, reflecting selection of patients in whom the risks of PCI were offset by the severity of the coronary involvement. He concludes: 'Excellent outcomes for most conservatively

Division of Cardiology, University of Washington, Seattle, Washington, USA

Correspondence to Professor Catherine M Otto, Division of Cardiology, University of Washington, Seattle, WA 98195, USA; cmotto@uw.edu
$A$
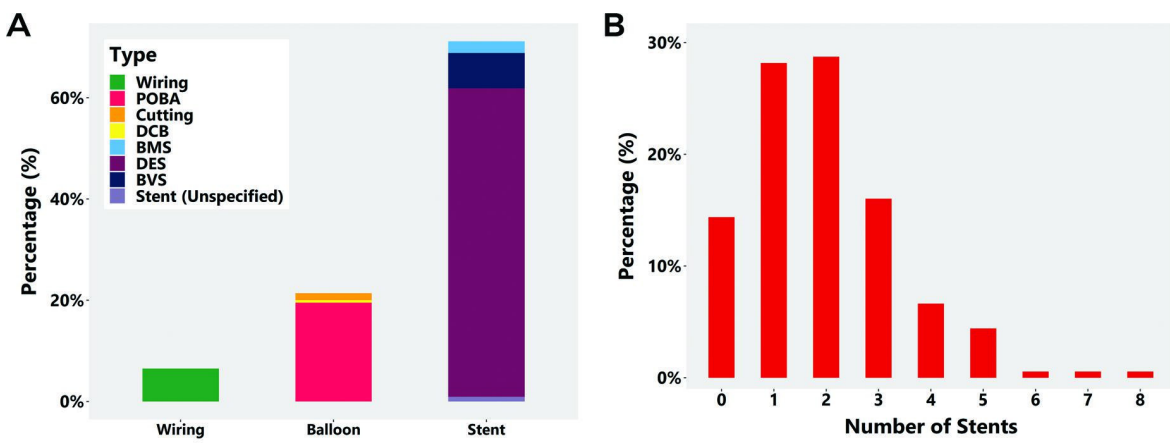

C

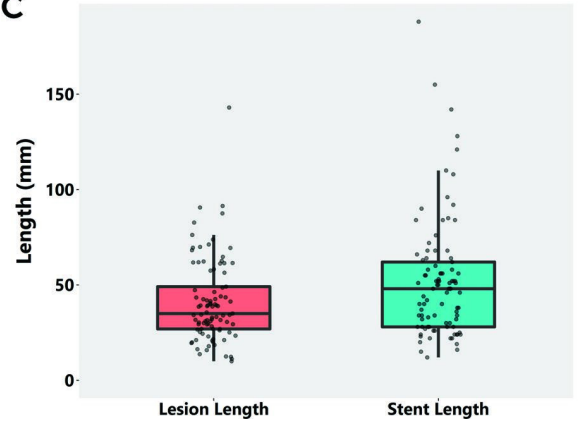

D

Stented Syntax Segments

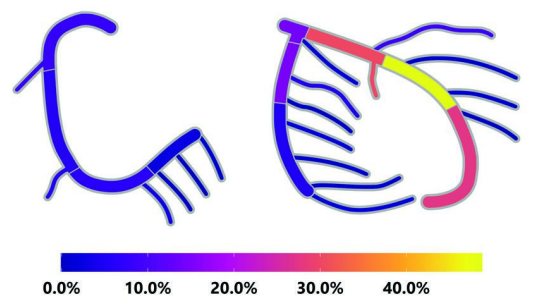

Figure 1 Details of the $P C I$ procedure in SCAD intervention patients $(n=215)$ : $(A)$ interventional strategy, (B) number of stents deployed, (C) stent length compared with lesion length and (D) coronary heat map of stented AHA coronary segments. AHA, American Heart Association; PCl, percutaneous coronary intervention; SCAD, spontaneous coronary artery dissection.

managed patients suggest our default should be to 'do no harm'. But, for an important minority where intervention is deemed a true necessity—complete vessel occlusion, haemodynamic instability-we ought to take courage that there is scope 'to do good'.'

The effectiveness of angiotensin receptor-neprilysin inhibitor (ARNI) treatment versus renin-angiotensin system (RAS) blockade alone was evaluated by Desai and colleagues ${ }^{3}$ in a cohort of over 50000 older (average age 76 years) adults with heart failure due to reduced ejection

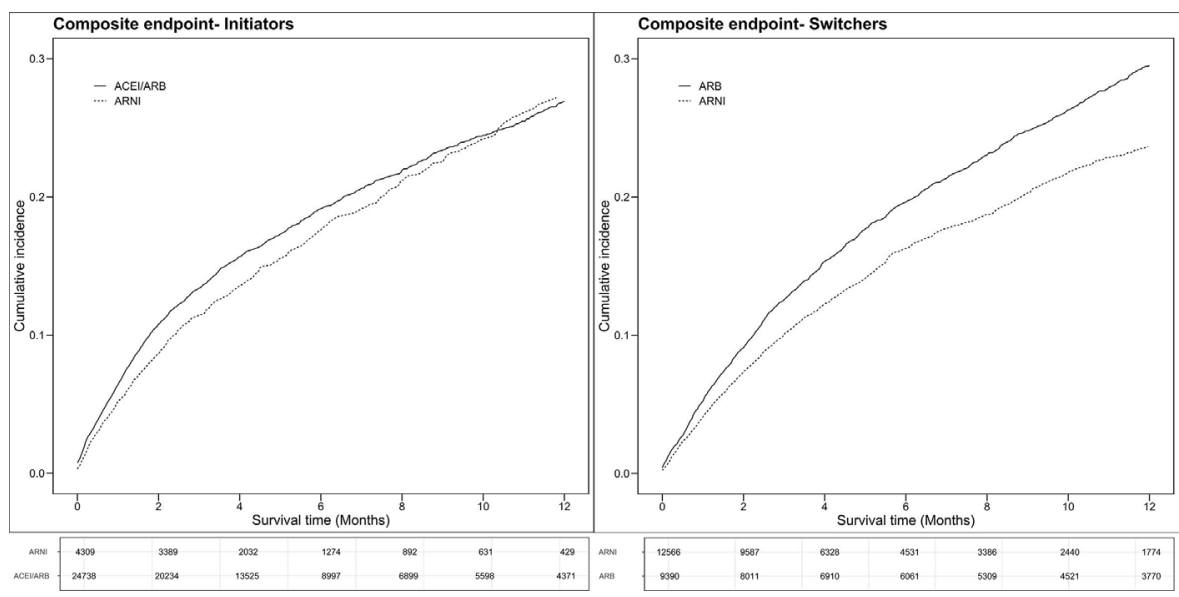

Figure 2 Cumulative incidence of individual endpoints of worsening heart failure and all-cause mortality after propensity score weighting in patients treated with angiotensin receptor-neprilysin inhibitor (ARNI) or renin-angiotensin system blockade alone. Numbers in the table below the plot represent populations at risk under as-treated follow-up. ARB, angiotensin receptor blocker. 


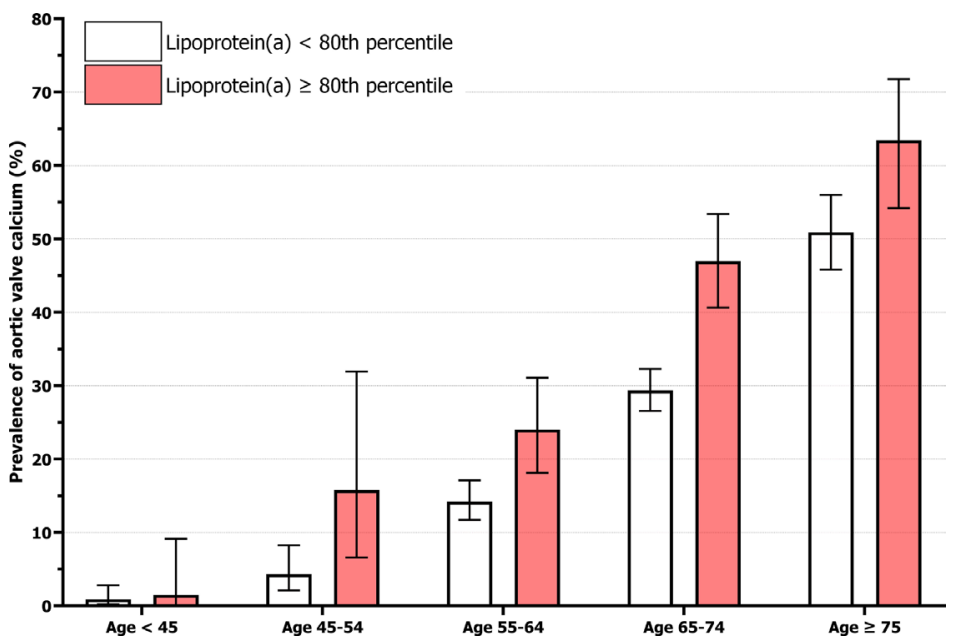

Figure 3 Prevalence of aortic valve calcium stratified by age and lipoprotein(a). Aortic valve calcium was defined as an aortic valve Agatston score $>0$. The prevalence of aortic valve calcium for lipoprotein(a) above and below the 80th percentile $(47.7 \mathrm{mg} / \mathrm{dL})$, respectively, was 1 of 67 $(1.5 \%)$ vs 3 of $339(0.9 \%)$ for ages below 45,6 of $38(15.8 \%)$ vs 9 of $210(4.3 \%)$ for ages $45-54$, 43 of $179(24.0 \%)$ vs 96 of $676(14.2 \%)$ for ages 55-64, 116 of $247(47.0 \%)$ vs 295 of 1005 (29.4\%) for ages 65-74 and 78 of $123(63.4 \%)$ vs 197 of 387 (50.9\%) for ages 75 and over. Error bars indicate $95 \%$ Cls. fraction (HFrEF). Patients treated with an ARNI, compared with RAS blockade alone, had a lower 1-year cumulative incidence of the composite outcome of worsening HF events or all-cause mortality both those in whom ARNI was the initial treatment ('initiators', $27.2 \%$ vs $26.9 \%$ ) and among those who switched from RAS blockade to ARNI treatment ('switchers', $23.8 \%$ vs $29.7 \%$ ), even after adjustment for numerous pre-exposure characteristics (figure 2).

In the accompanying editorial, Griffin and $\mathrm{Cheng}^{4}$ put this real-world data into the context of previous clinical trials. For example, mean age in the current study was older compared with clinical trials

(76 vs 64 years), more women (33\% vs $21 \%)$ and black patients (11\% vs $5 \%)$ were included, and a sizeable number (12\%-14\%) had chronic kidney disease. They also point out that the 'switcher' group likely represents more chronic HF compared with the 'initiator' group, possibly explaining the apparent greater effect in the "switcher group". However, in the 'real world' most patients eligible for an ARNI still do not receive this mediation despite guideline recommendations that 'ARNI is now indicated in patients with chronic heart failure regardless of ejection fraction', highlighting the barriers to uptake including lack of physician education, concerns about safety of
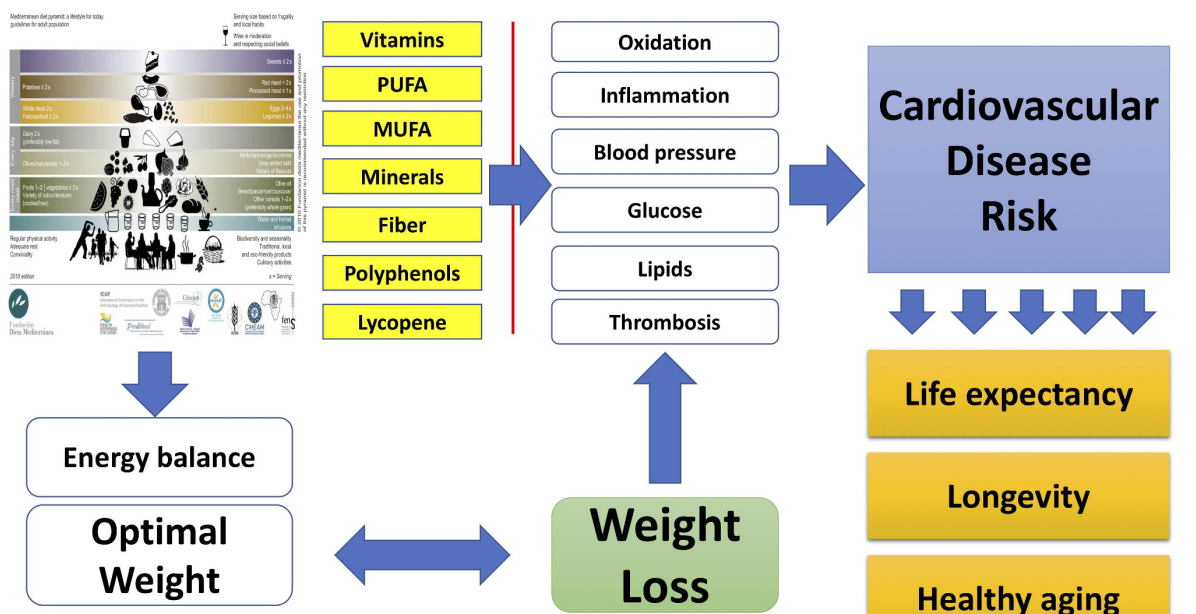

Life expectancy

Longevity

Healthy aging

Figure 4 Mediterranean lifestyle, weight and energy balance, in relation to cardiovascular disease risk. MUFA, monounsaturated fatty acids; PUFA, polyunsaturated fatty acids.

switching medications, particularly the effects on blood pressure, and the cost of the drug for patients. Griffin and Cheng conclude 'As we continue to evaluate efficacy of new indications for ARNI therapy and the focus turns to HF with mid-range and preserved EF, we must continue to improve morbidity and mortality in HFrEF by making it available to those in whom it already has proven benefit.'

In another interesting paper in this issue of Heart, Kaiser and colleagues ${ }^{5}$ looked at the association between serum lipoprotein(a) $(\mathrm{Lp}(\mathrm{a}))$ levels and the prevalence and quantity of aortic valve calcium (AVC), measured by cardiac CT, in 2412 participants in the population-based Rotterdam Study as well as 859 healthy individuals from the Amsterdam University Medical Centers (UMC) outpatient clinics. In both cohorts, higher Lp(a) levels were associated with higher valve calcium scores although the prevalence of AVC was $33.1 \%$ in the Rotterdam Study versus $5.4 \%$ in the Amsterdam UMC cohort (figure 3).

Tzolos and Dweck ${ }^{6}$ succinctly summarise the key finding of this study: 'Once again, Lp(a) appears associated with incident AS and the initiation phase of the disease. Interestingly, there appears to be a threshold effect underlying this association around the 80 th percentile $(47.7 \mathrm{mg} /$ dL).' They emphasise that this data is important because 'In order to adequately power future randomised controlled trials, we need to target the patients who might benefit most from an intervention. This study suggests that $\mathrm{Lp}(\mathrm{a})$ lowering should target patients above the 80th $\mathrm{Lp}$ (a) percentile $(\geq 50 \mathrm{mg} / \mathrm{dL})$ independent of their age.' However, 'Ultimately, doubleblinded randomised controlled trials are required to assess whether $L p(a)$ lowering in patients with aortic valve calcification can slow disease progression and improve clinical outcomes.'

The Education in Heart article in this issue reviews the challenging issue of weight loss through lifestyle changes for primary prevention of cardiovascular disease. Yannakoulia and Panagiotakos ${ }^{7}$ acknowledge the difficulty in helping patients improve dietary patterns and engage in regular exercise, as well as the high prevalence of weight regaining even when initial weight loss efforts are successful. The Mediterranean diet/lifestyle has the most scientific evidence establishing benefits and as summarised in the article (figure 4).

The Cardiology in Focus article in this issue of Heart provides useful guidance for medical students, residents or cardiology 
trainees interested in pursing a career in preventative cardiology. 8

Funding The authors have not declared a specific grant for this research from any funding agency in the public, commercial or not-for-profit sectors.

Competing interests None declared.

Patient and public involvement Patients and/ or the public were not involved in the design, or conduct, or reporting, or dissemination plans of this research.

Patient consent for publication Not required.

Provenance and peer review Commissioned; internally peer reviewed.

(C) Author(s) (or their employer(s)) 2021. No commercia re-use. See rights and permissions. Published by BMJ.

\section{Check for updates}

To cite Otto CM. Heart 2021;107:1357-1359.

Heart 2021:107:1357-1359.

doi:10.1136/heartjnl-2021-320055

ORCID iD

Catherine M Otto http://orcid.org/0000-0002-05279392

\section{REFERENCES}

1 Kotecha D, Garcia-Guimaraes M, Premawardhana D. Risks and benefits of percutaneous coronary intervention in spontaneous coronary artery dissection. Heart 2021;107:1398-406.

2 Adamson PD. Spontaneous coronary artery dissection: to do good or to do no harm? Heart 2021;107:1362-3.
3 Desai RJ, Patorno E, Vaduganathan M, et al. Effectiveness of angiotensin-neprilysin inhibitor treatment versus renin-angiotensin system blockade in older adults with heart failure in clinical care. Heart 2021;107:1407-16.

4 Griffin J, Cheng R. Real-World utilisation of angiotensinneprilysin inhibitors in older adults with heart failure. Heart 2021:1364-6.

5 Kaiser Y, Singh SS, Zheng KH, et al. Lipoprotein(a) is robustly associated with aortic valve calcium. Heart 2021;107:1422-8.

6 Tzolos E, Dweck MR. Threshold effect for lipoprotein(a) in aortic stenosis. Heart 2021;107:1367-8.

7 Yannakoulia M, Panagiotakos D. Weight loss through lifestyle changes: impact in the primary prevention of cardiovascular diseases. Heart 2020;107:1429-34.

8 German CA, Shapiro MD. Roadmap to a career in preventive cardiology. Heart 2021;107:1435-6. 